\title{
AUTO-EFICACIA DOCENTE, MOTIVACIÓN INTRÍNSECA Y EXPECTATIVA DE RESULTADO DEL ALUMNADO
}

\section{PROFESSORIAL SELF-EFFICACY, INTRINSIC MOTIVATION, AND PERFORMANCE EXPECTATION OF STUDENTS}

Joel Andrés Revelli Galarza, Pablo Gutiérrez Sedeño, Federico del Castillo Fernández, María Centeno Mudarra, Alejandro Vinuesa González, Basma Belcaid y María Teresa Andrade Robles. Universidad de Granada

\section{RESUMEN}

La Teoría Social-Cognitiva de Bandura da gran importancia a la auto-eficacia como método regulador del aprendizaje y sus diferentes elementos, como la motivación y la expectativa de resultado. Por ello, el objetivo de este estudio es determinar en qué grado se relacionan ambos constructos en un contexto universitario y como la motivación intrínseca afecta la expectativa de resultado en alumnos. Para ello se han pasado cuestionarios de auto-eficacia a tres profesores, y cuestionarios de motivación intrínseca sus respectivos alumnos. No se han podido concretar los resultados esperados en la mayoría de las comparaciones en cuanto a relación autoeficaciamotivación, pero sí se ha visto una relación observable entre motivación y expectativa de resultado del alumnado. Se puede concluir que la alta auto-eficacia docente es un factor decisivo en el aprendizaje.

Palabras clave: motivación, papel del docente, interés (aprendizaje), rendimiento de la educación; eficacia del docente.

\begin{abstract}
Bandura's Social Cognitive Theory gives great importance to self-efficacy as regulator for learning and its different elements, like motivation and performance expectations. And so, the goal of this work is to determine the degree of connection between both constructs in a university context and how intrinsic motivation influences performance expectations in students. For this purpose, self-efficacy tests have been used for three professors and an intrinsic motivation test for their respective alumni. The hypothesized results eluded the investigation in most of the comparisons concerning self-efficacy and motivation, but this work did yield the expected results in an observable relationship between motivation and performance expectations of alumni. It can be ascertained that high professorial self-efficacy is a decisive factor in learning.
\end{abstract}

Key words: motivation, teacher role, interest (learning), educational output; teacher effectiveness.

\section{Introducción}

Auto-eficacia: La forma en la que los profesores consideran sus propias capacidades y limitaciones tiene un efecto importante en cómo se desenvolverán en su trabajo y cómo afectarán a sus alumnos. Estudiar esta relación es vital si hemos de entender cómo la relación entre profesor y alumno afecta a estos últimos, y nos da una visión mucho más dinámica de factores interpersonales a tomar en cuenta en el proceso de aprendizaje. 
Dentro del constructo teórico cognitivo-social de Albert Bandura se le da mucha importancia a los procesos auto-reflexivos y auto-regulativos. Bandura se aleja del concepto de persona reactiva y dependiente de las circunstancias (1986), siendo la auto-eficacia uno de los núcleos de su teoría reivindicativa de la importancia de cogniciones mediadoras. Define este concepto como las creencias de la gente sobre sus capacidades para producir niveles designados de rendimiento que ejerciten influencia sobre eventos que afectan a sus vidas (Bandura, 1994). Los trabajos de Bandura han defendido reiteradamente que la auto-eficacia influye en nuestra conducta e incluso nuestro éxito o fracaso, así como los trabajos de Pajares (1997), de Pintrich y Schunk (1996) y Gónzalez (2010).

Muchos estudios y artículos se han hecho para verificar los efectos de la auto-eficacia. Nosotros nos centraremos en los aspectos educacionales del constructo, concretamente su relación con los educadores y sus implicaciones en clase. Por ejemplo, se observó que la auto-eficacia percibida por profesores determina sus conductas particulares de enseñanza (Woolfolk y Hoy, 1990) y correlacionan positivamente con la satisfacción de su trabajo, mostrando más compromiso (Trentham et al. 1985). Así mismo, maestros con alta auto-eficacia tienden a persistir ante situaciones de fracaso en la enseñanza y a innovar en métodos de instrucción (Gibson y Dembo, 1984).

Todas las consecuencias de la auto-eficacia descritas arriba tienen efectos sobre la clase en su conjunto. La auto-eficacia del profesor no solo influencia sus creencias y motivación, sino que también tiene un rol fundamental en la educación de los alumnos. Parece ser que una buena auto-eficacia en el profesor promueve un mejor rendimiento académico estudiantil (Ashton y Webb, 1986; Moore y Esselman, 1992), y mejora la percepción de su propia auto-eficacia (Anderson et al. 1988; Midgely et al. 1989). La confianza del profesor en sus capacidades también promueve la confianza hacia las capacidades de sus alumnos: menos alumnos serán recomendados para educación especial si su profesor es auto-eficaz, y recibirán más ayuda y menos crítica cuando fallan (Gibson y Dembo, 1984). También parece que alumnos con profesores de alta auto-eficacia son incitados más a menudo a completar tareas de mayor dificultad y a desarrollar un entendimiento más profundo sobre la tarea, gracias a que el profesor tiene más recursos cognitivos y emocionales disponibles, quizá por temerle menos al conflicto con estudiantes y a tomar mayores riesgos intelectuales e interpersonales (Woolfolk Hoy y Davis, 2005). Las tasas de suspensiones y abandonos del alumnado también son menores si el profesorado suele tener alta auto-eficacia (Goddard, 2001). Estos son solo algunos de los muchos estudios llevados a cabo que indican grandes beneficios de una buena auto-eficacia en los educadores, beneficios que se extienden a los alumnos. De estos, el que nos interesa discutir es el efecto que tiene sobre la motivación estudiantil, que consideramos es la raíz de los cambios positivos en ellos.

Motivación: Muchos autores coinciden en que la motivación se define como un conjunto de procesos implicados en la activación, dirección y persistencia de la conducta (Beltrán, 1993; Bueno, 1995; McClelland, 1989). Este constructo se compone de la motivación extrínseca, que es estimulada por incentivos externos ya sean auto-administrados o administrados por otros, y la motivación intrínseca, que es estimulada por la tarea en sí y carente de incentivos externos (Ryan y Deci, 2002), de las cuales nos centraremos en esta última.

La motivación intrínseca es importantísima en el estudiante. Afrontar una tarea por el hecho de aprender en sí, y no por una "recompensa", libera al estudiante de las presiones de la competencia entre pares y del "conservadurismo académico", o sea, ir a lo seguro y a lo fácil con tal de sacar buena nota y no arriesgarse o ampliar e ir más allá de lo requerido (Elliot, 1999; Pajares, Britner y Valiente, 1996). Trabajar 
intrínsecamente motivado posibilita incrementar, capacidades, competencias y el disfrute de uno en la tarea, y por ende, su rendimiento académico (Dweck y Elliot, 1883; Deci y Ryan, 1985; Alonso Tapia, 1997), logrando superar aburrimiento y ansiedad (Csikszentmihalyi, 1975), y buscando soluciones y ayuda por uno mismo (Jagacinsky, 1992). Plantearse una tarea como un proyecto personal es una consecuencia de la motivación intrínseca que genera efectos muy positivos en la forma en la que el estudiante afronta la tarea y a su educación.

El papel del profesor tiene un efecto positivo sobre el rendimiento del alumno asociado a la motivación intrínseca (Boza Carreño y Toscano Cruz, 2012). Dada la estrecha concordancia teórica que tiene la auto-eficacia percibida del profesor con la motivación y rendimiento del estudiante, demostrada en numerosos estudios que relacionan la auto-eficacia del educador con varios y diversos factores componentes o consecuentes del constructo de la motivación, nuestra hipótesis se basa en el efecto de la auto-eficacia percibida del profesor por parte del alumno sobre la motivación intrínseca de este en particular, y se operativiza de la siguiente forma: La auto-eficacia percibida por los profesores se relacionará positivamente con la motivación intrínseca de sus estudiantes.

También queremos comprobar que una alta motivación intrínseca mejora la expectativa de nota, que es un componente de la auto-eficacia del alumno en esa asignatura.

\section{Método}

\section{Participantes}

El número total de participantes en este estudio ha sido de 101,3 profesores universitarios de Psicología y 98 de sus alumnos. 45 son alumnos de $2^{\circ}$ de grado, 31 alumnos de $3^{\circ}$ de grado y 21 alumnos de $4^{\circ}$ de licenciatura.

\section{Instrumentos}

Auto-eficacia de los profesores: Para medir la auto-eficacia se ha utilizado una adaptación al español de la Teacher's sense of efficacy Scale elaborado por Tshannen-Moran y Woolfolk (2001). El test consta de 44 items que se pueden subdividir en 3 escalas más la escala global de auto-eficacia. (a) eficacia percibida para optimizar la propia instrucción, (b) eficacia percibida para gestionar el aula y (c) eficacia para implicar al estudiante en el aprendizaje. La escala de Tshannen-Moran y Woolfolk ha mostrado validez y una gran fiabilidad tanto de la escala global como de las tres subescalas (Klassen et al., 2009). En el presente trabajo utilizaremos únicamente la escala global.

Motivación intrínseca de alumnos: Para la motivación hemos utilizado el test psicométrico de motivación intrínseca (Vilchez, 2008) que consta de 40 items validados por un grupo de expertos.

\section{Procedimiento}

Los datos han sido recogidos en la facultad de Psicología de Granada. Tanto la escala de auto-eficacia a los profesores como el test de motivación fueron realizados en el horario de clases habitual 


\section{Resultados}

En la tabla 1 se presentan los datos descriptivos de los grupos de alumnos con profesor de alta auto-eficacia (grupo alta auto-eficacia), con profesor de auto-eficacia media (grupo media auto-eficacia) y con profesor de baja auto-eficacia (grupo baja auto-eficacia).

\begin{tabular}{lllll}
\hline & Grupo auto-eficacia & $\mathrm{N}$ & Media & DT \\
\hline \multirow{2}{*}{ MOTIVACION } & Alta & 45 & 3.76 & .36 \\
& Media & 31 & 3.56 & .39 \\
& Baja & 21 & 3.53 & .34 \\
\hline
\end{tabular}

Hipótesis 1: Con el objetivo de analizar si existían diferencias en la motivación y en la nota esperada en los participantes en función del grupo de alta eficacia, media eficacia y baja se han realizado diferentes pruebas $t$ de student para muestras independientes (véase gráficas 1 y 2 )

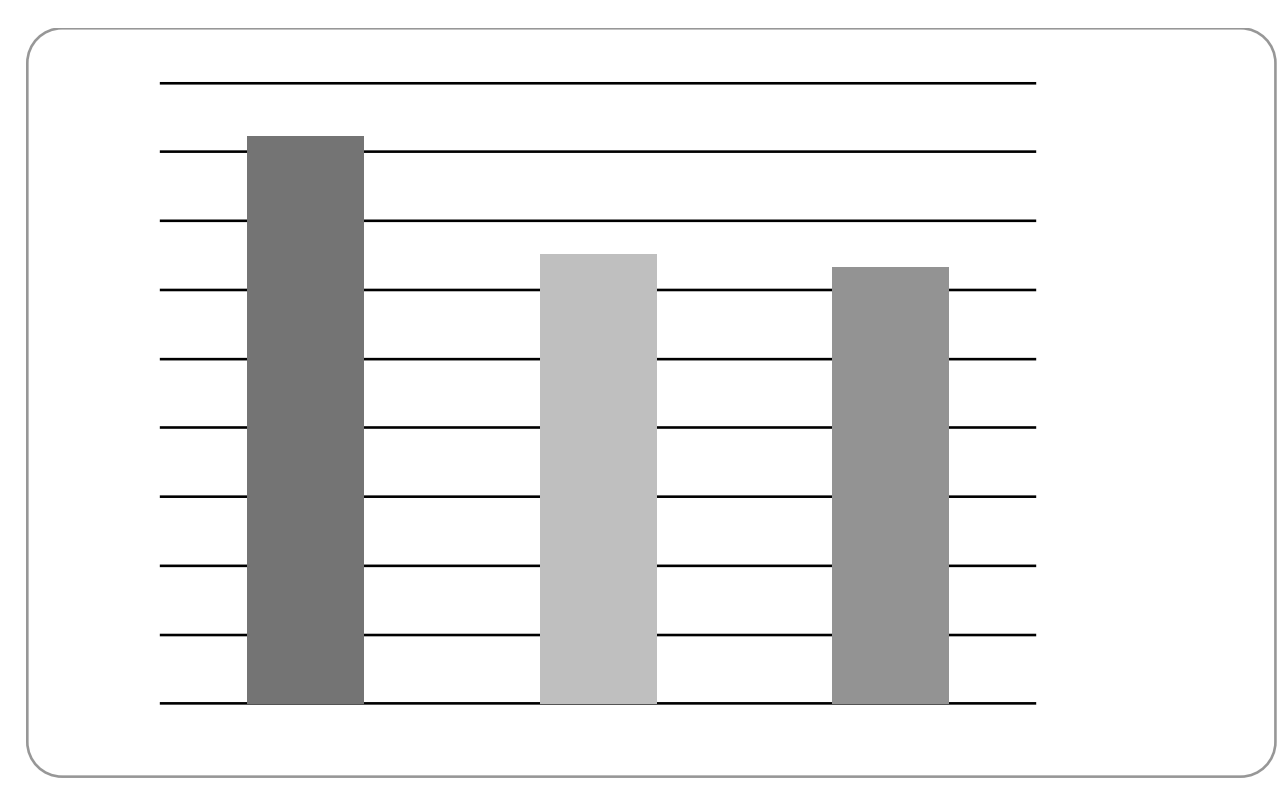

Gráfica 1. Puntuaciones obtenidas por los participantes en la nota esperada en función de la alta, media y baja autoeficacia del profesor 


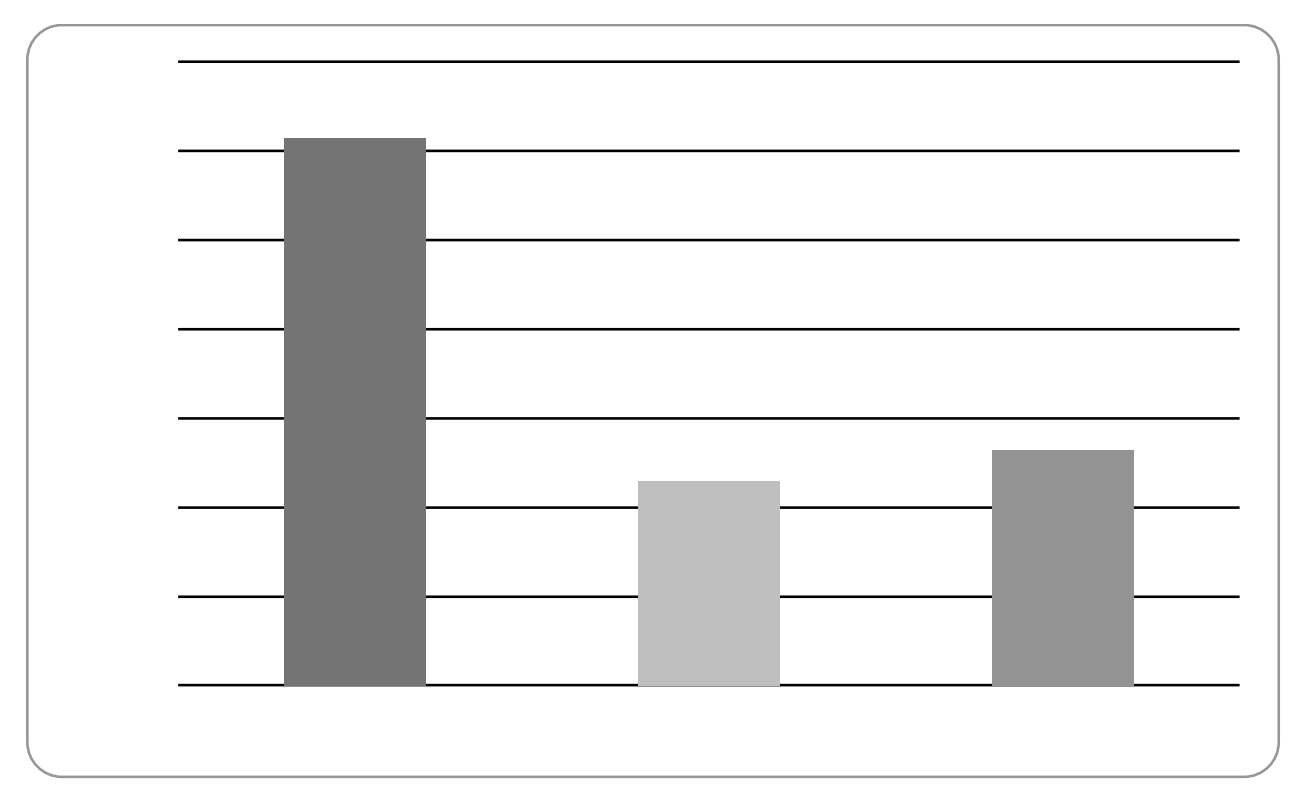

Gráfica 2. Puntuaciones obtenidas por los participantes en la motivación en función de la alta, media y baja autoeficacia del profesor

Tras comparar los grupos se encontraron diferencias significativas al estudiar el grupo de Alta Autoeficacia (Grupo1) con el grupo de Media Autoeficacia (Grupo 2) tanto en motivación $\mathrm{t}(73)=2.18, \mathrm{p}<.05$ como en la nota esperada $\mathrm{t}(73)=5.91, \mathrm{p}<.05$.

Hipótesis 2: Con el objetivo de analizar las relaciones existentes entre la motivación y la expectativa de la nota (NParcial) en los distintos grupos (alta eficacia, media eficacia y baja eficacia), se llevó a cabo un análisis de correlación mediante el coeficiente de correlación de Pearson (veáse Tabla 2).

Tabla 2. Correlaciones bivariadas entre las diferentes variables

$$
\text { Nota }
$$

Alta Eficacia $\quad$ Media Eficacia $\quad$ Baja Eficacia

$\begin{array}{llll}\text { Motivación } & .416^{* *} & .165 & -.696^{* *}\end{array}$

Nota: ${ }^{*} p<.05^{* *} p<.01^{* * *} p<.001$

Como se puede observar, existe una correlación significativa y positiva entre la motivación y la nota en los grupos de alta autoeficacia, de modo que a mayor motivación, una mayor motivación. Los resultados también mostraron una correlación significativa negativa en el grupo de baja eficacia, de modo que en el grupo de profesores con baja eficacia, una mayor motivación se relaciona con una menor nota esperada. Para el grupo de media eficacia, no existe una relación significativa entre la motivación y el rendimiento esperado. 


\section{Discusión}

Primero de todo, hay que aclarar: ¿Por qué nos concentramos en la motivación intrínseca y no en la extrínseca, o en ambas? La razón es que la auto-eficacia del profesor no puede definirse como una "meta" o recompensa externa. Su eficacia funciona como un medio de apoyo al estudiante y no como un fin. Pues, aunque un profesor eficaz sí logre mejorar la motivación extrínseca en el alumno, el efecto no sería puro ya que, además de la auto-eficacia percibida en el educador, también se requeriría de un incentivo externo para argumentar causalidad en el cambio del alumno, causalidad que sería compartida (entre la auto-eficacia percibida del profesor y la influencia del incentivo) y posiblemente sesgada, cosas que no discutiremos en este trabajo. La motivación intrínseca no tiene este problema y se puede medir el efecto sobre el alumno de la auto-eficacia profesoral percibida de forma más directa, sin tanta contaminación metodológica.

En este trabajo se ha pretendido encontrar una relación positiva entre la auto-eficacia percibida del profesor y la motivación intrínseca de sus estudiantes. Aunque existe una tendencia de la motivación intrínseca a desarrollarse cuanto mayor sea la auto-eficacia del educador, los resultados no indican que esta diferencia sea suficientemente significativa en los grupos de media y baja auto-eficacia. Hay dos razones principales por las que creemos que obtuvimos esta baja significación: la primera es la poca diferencia en la puntuación de auto-eficacia de los profesores, es decir, no diferían lo suficiente como para reflejarse en las puntuaciones de motivación estudiantil. La segunda razón es la falta de control en las variables situacionales externas que afectan a la motivación, como puede ser la dificultad dispar entre asignaturas impartidas. Mucho se han estudiado los efectos de la dificultad de una tarea sobre la motivación (Csikszentmihalyi, 1990). El diferente contenido de las clases puede haber afectado los resultados. Otras investigaciones con grupos más similares y profesores más dispares podrían mejorar el diseño experimental y dar resultados más significativos.

Los resultados de cada comparación particular de grupos podrían indicar que la relación entre la auto-eficacia y la motivación solo se daría en el supuesto de que el profesor tenga una alta auto-eficacia. Estos resultados se podrían relacionar con las conclusiones aportadas en otro estudio en el que se afirmaba que los profesores con una autoeficacia alta le dan más importancia a aspectos extrínsecos como el sentido del deber o disfrutar con el aprendizaje del alumno (Blas, Núñez, Rodríguez, Rosario, \& Valle, 2009). Esta relación no se observa en los grupos con profesores de media y baja auto-eficacia. Esto puede deberse a que los estudiantes de estos educadores buscan otras fuentes de motivación externas, mostrando que no deriva en efectos negativos sobre la motivación per se, y resalta los efectos positivos de una alta autoeficacia del profesorado.

Los resultados de la segunda hipótesis, no obstante, corroboran investigaciones previas que defendían la motivación como un factor determinante en las expectativas de resultado (Bandura, 1977). Sin embargo, en uno de los grupos esta correlación no aparece como significativa. Esto puede deberse a los extremos de dificultad (muy alta o muy baja) percibida de la asignatura en concreto y hace que la expectativa de nota sea independiente de la motivación del alumnado.

Ciertamente, el estudio de la auto-eficacia ha dado fruto, pero el concepto no está exento de debate. Pajares (1997) defiende que la auto-eficacia debe ser estudiada en contexto, y relacionada a conductas específicas. Por otro lado, se pone en tela de juicio la validez de las puntuaciones obtenidas en los instrumentos de medición primarios de la auto-eficacia (Coladarci \& Fink, 1995; Guskey \& Passaro, 1994). Una 
tercera vía de estudio sería entonces la revisión del constructo en su totalidad, con las posibles implicaciones que esto tendría para la teoría social-cognitiva de Bandura.

A pesar de los pormenores del diseño experimental, creemos que se observan pautas prometedoras que explotar y expandir en el futuro mediante otras investigaciones. Una línea de investigación interesante sería contrastar los efectos de los distintos tipos de auto-eficacia (cultural, emocional, cognitiva y conductual) en los diferentes tipos de motivación (de logro, de afiliación, de dominio, etc.). Otro posible estudio podría adentrarse en los factores que influencian a la auto-eficacia profesoral, como por ejemplo rendimiento logrado anteriormente, experiencia vicaria, simbolización imaginativa, persuasión verbal y activación emocional y fisiológica. También se podría relacionar la autoeficacia con las estrategias de aprendizaje ya que se ha demostrado que profesores que utilizan estrategias enfocadas en el aprendizaje influyen mñas positivamente sobre el aprendizaje de sus alumnos (Fernández March, Garfella Esteban, Gargallo López, \& Pérez Pérez, 2010). Esto abriría las puertas a un entrenamiento docente mucho más adecuado y enfocado al incremento motivacional del alumnado.

\section{BIBLIOGRAFÍA}

Alonso Tapia, J. (1997). Motivar para el aprendizaje. Teoría y Estrategias. EDEBE, Barcelona.

Ashton y Webb (1986) Making a Difference: Teacher Efficacy and Student Achievement. Monogram. White Plains, NY: Longman.

Bandura, A. (1977). Self-efficacy: toward a unifying theory behavioral change. Psychogical Review, 84(2), 191-215.

Bandura, A. (1986). The explanatory and predictive scope of self-efficacy theory. Journal of Social and Clinical Psychology, 4 (3), 359-373.

Bandura A. (1994). Self-efficacy. In V.S. Ramachandran (Ed.) Encyclopedia of human behavious (Vol.4, pp.71-81). New York: Academic Press.

Beltrán, J. (1993). Procesos, estrategias y técnicas de aprendizaje. Madrid: Síntesis. S.A.

Blas, R., Núñez, J. C., Rodríguez, S., Rosario, P., \& Valle, A. (2009). Auto-eficacia Docente, Motivación del Profesor y Estrategias de Enseñanza. Escritos de Psicología, 3(1), 1-7.

Boza Carreño, A., y Toscano Cruz, M. d. (2012). Motivos, actitudes y estrategias de aprendizaje: Aprendizaje motivado en alumnos universitarios. Profesorado , 16 (1).

Bueno, J. A. (1995): Motivación y aprendizaje. En Beltrán y Bueno: Psicología de la educación. Barcelona: Marcombo, pp. 227-255.

Coladarci, T., y Fink, D. R. (1995). Correlations among measures of teacher efficacy: Are they measuring the same thing? Paper presented at the annual meeting of the American Educational Research Association, San Francisco.

Csikszentmihalyi, M. (1975). Beyond Boredom and anxiety. Jossey Bass. San Francisco. 
Csikszentmihalyi, M. (1990). Flow: The Psychology of Optimal Experience. New York: Harper-Row.

Deci, E. L., y Ryan, R. M. (1985). Intrinsic motivation and self-determination in human behaviour. New York: Plenum.

Deci, E. L., y Ryan, R. M. (1985).The general causality orientations scale: Selfdetermination in personality. Journal of Research in Personality, 19, 109-134.

Dweck, C.S. \& Elliot, E.s. (1983). Achievement motivation. En E.M. Hetherington (ed.) Socialization, personality and social development. Wiley \& sons, N.Y.

Elliot, A. J. (1999) Approach and avoidance motivation and achievement goals, Educational Psychologist, 34, 169-189.

Fernández March, A., Garfella Esteban, P. R., Gargallo López, B., \& Pérez Pérez, C. (2010). Modelos de enseñanza y aprendizaje en la universidad. Seminario Interuniversitario de Teoría de la Educación. Madrid.

González, M. L. (2010). Autoeficacia percibida y desempeño académico en estudiantes universitarios. Acceso y Permanencia en una educacion de calidad. Buenos Aires.

Gibson, S., \& Dembo, M.H. (1984). Teacher efficacy: A construct validation. Journal of Educational Psychology, 76, 569-582.

Goddard, R. D., \& Goddard, Y. L. (2001). A multilevel analysis of the relationship between teacher and collective efficacy in urban schools. Teacher and Teacher Education, 17, 807-818.

Guskey, T., \& Passaro, P. (1994). Teacher efficacy: A study of construct dimensions. American Educational Research Journal, 31, 627-643.

Jagacinski, C.M. (1992). The effects of task involvement and ego involvement and achievement-related cognition and behaviours. En D.H. Schunk y J.L. Meece (Eds.) Students' perceptions in the classroom. (pp. 307-326) Laurens Erlbaum. Hillsdale, NJ.

Klassen, R.M., Bong, M., Usher, E.L., Chong, W.H., Huan, V.S., Wong, I.Y.F. y Georgiou, T. (2009). Exploring the validity of a teachers' self-efficacy scale in five countries. Contemporary Educational Psychology, 34, 67-76.

Midgely, C., Feldlaufer, H., \& Eccles, J.S (1989). Change in teacher efficacy and student self- and task-related beliefs in mathematics during the transition to junior high school. Journal of Educational Psychology, 81 (2), 247-258.

Pajares F. (1997). Current directions in self-efficacy research. In M. Maehr \& P.R. Pintrich (Eds.). Advances in motivation and achievement (Vol.10, pp. 1-49). Greenwich, CT: JAI Press.

Pajares, F.; Britner, S. L. y Valiente, G. (2000) Relation between achievement goals and self-beliefs of middle school students in writing and science, Contemporary Educational Psychology, 25, 406-422.

Pintrich, P. \& Schunk, D. (1996). Motivation in Education: Theory, Research \& Applications, Ch. 3. Englewood Cliffs, NJ: Prentice-Hall. 
Ryan, R. M., \& Deci, E. L. (2002). An overview of self-determination theory. In E. L. Deci \& R. M. Ryan (Eds.), Handbook of self-determination research (pp. 3-33). Rochester, NY: University of Rochester Press.

Trentham, L., Silvern, S., \& Brogdon, R., (1985). Teacher efficacy and teacher competency ratings. Psychology in the Schools, 22 (3), 343-352.

Tschannen-Moran M. y Woolfolk, A. (2001). Teacher efficacy: capturing an elusive construct. Teaching and Teacher Education, 17, 783-805.

Vilchez, A. (2008). Test psicométrico para medir el grado de motivación intrínseca. Extraído el 27-5-2013 de: http://es.scribd.com/doc/17379211/Test

Woolfolk, A. E. \& Hoy, W. K. (1990). Prospective teachers' sense of efficacy and beliefs about control. Journal of Educational Psychology, 82(1), 81-91.

Woolfolk Hoy, A., \& Davis, H. (2005). Teachers' sense of efficacy and adolescent achievement. In T. Urdan \& F. Pajares (Eds.), Adolescence and education: Vol. 5: Selfefficacy beliefs during adolescence (pp. 117-137). Greenwich, CT: Information Age. 